\title{
Analytic Univalent Functions Defined by Generalized Discrete Probability Distribution
}

\author{
Abiodun Tinuoye Oladipo \\ Department of Pure and Applied Mathematics, Ladoke Akintola University of Technology, \\ Ogbomoso, P.M.B. 4000, Ogbomoso, Oyo State, Nigeria \\ e-mail: atoladipo@lautech.edu.ng
}

\begin{abstract}
The close-to-convex analogue of a starlike functions by means of generalized discrete probability distribution and Poisson distribution was considered. Some coefficient inequalities and their connection to classical Fekete-Szego theorem are obtained. Our results provide strong connection between Geometric Function Theory and Statistics.
\end{abstract}

\section{Introduction}

Let $A$ denote the class of analytic functions $f$ in the unit disk $E=\{z:|z|<$ $1\}$. We denote by $S$ the subclass of $A$ of analytic univalent functions $f$ with normalization condition $f(0)=f^{\prime}(0)-1=0$ in $E$.

We recall here that for any $f \in A$ the power series representation is given by

$$
f(z)=z+\sum_{k=2}^{\infty} a_{k} z^{k} .
$$

Let $S^{*}(0) \subset S$ be the family of starlike functions of order zero defined by the geometric condition

$$
\operatorname{Re} \frac{z f^{\prime}(z)}{f(z)}>0, z \in E
$$

Received: July 15, 2020; Accepted: August 21, 2020

2010 Mathematics Subject Classification: 30C45, 30C50.

Keywords and phrases: analytic, univalent, Poisson, discrete, distribution series, probability, Fekete-Szego, coefficient.

${ }^{*}$ Corresponding author 
A function $f \in A$ is said to be convex if it satisfies the condition

$$
\operatorname{Re}\left\{1+\frac{z f^{\prime \prime}(z)}{f^{\prime}(z)}\right\}>0, z \in E .
$$

We denote by $C(0)$ the class of convex functions of order zero.

For easy handling, we also use the notations $S^{*}=S^{*}(0)$ and $C=C(0)$.

A function $f \in A$ is said to be close-to-convex if there exists $\rho \in\left(-\frac{\pi}{2}, \frac{\pi}{2}\right)$ and a function $g \in S^{*}$ such that

$$
\operatorname{Re}\left\{e^{i \rho} \frac{z f^{\prime}(z)}{g(z)}\right\}>0, z \in E
$$

Whenever $\rho=0$ the class of close to convex function is denoted by $K$ and $S^{*} \subset$ $K \subset A$.

Here we recall the class of Caratheodory function $P$ which are analytic in $E$ and satisfy $p(0)=1$ and $\operatorname{Rep}(z)>0$ for $z \in E$ with series representation

$$
p(z)=1+\sum_{k=1}^{\infty} p_{k} z^{k},\left|p_{k}\right| \leq 2 .
$$

Let $f \in A$, then $f \in S_{\mu}^{*}$ if

$$
\left|\frac{z f^{\prime}(z)}{f(z)}-1\right|<1
$$

and if $f \in S_{\mu}^{*}$, then

$$
\left|a_{k}\right| \leq \frac{1}{k-1}, k \geq 2 .
$$

It is clear that $S_{\mu}^{*} \subset S^{*}$. The above class was introduced and investigated by Singh [9] (see also [10]).

Recently in [1], 2], [7] derivations of certain geometric properties were carried out to establish connections between Geometric Function Theory and Statistics by means of generalized discrete probability distribution.

Let $S$ denote the sum of the convergent series with representation

$$
S=\sum_{k=0}^{\infty} a_{k},
$$


where $a_{k} \geq 0$, for all $k \in N$. The generalized discrete probability distribution whose probability mass functions given as $p(k)=\frac{a_{k}}{S}, k=0,1,2, \ldots, p(k)$ is the probability mass function because $p(k) \geq 0$ and $\sum_{k} p_{k}=1$.

Furthermore, we let $\phi(x)=\sum_{k=0}^{\infty} a_{k} z^{k}$, then from $S=\sum_{k=0}^{\infty} a_{k}$ series $\phi$ is convergent for $|x|<1$ and $x=1$.

Of particular interest to the present investigation is the power series whose coefficient are probabilities of the generalized distribution of the form

$$
K_{\phi}(z)=z+\sum_{k=2}^{\infty} \frac{a_{k-1}}{S} z^{k}
$$

which was recently studied in [[1], [2], [7]].

The purpose of the present investigation is to derive a robust geometric properties that establish a more stronger connections between Geometric Function Theory and Statistics; and to further point out the consequential effects when the quantity $a_{n_{s}}$ is replaced by Poisson probability distribution. Our result is robust because obtaining sharp estimates for the coefficients represents a much more difficult problem most especially when to extend from starlikeness to close-to-convexity. To achieve this our method shall follow the pattern of that of Allu et al. [10].

Next we define the close to convex analogue of the class $S_{\mu}^{*}$ associated with generalized distribution as follows.

Definition 1.1. We say that $K_{\phi} \in K_{\mu}$ if $K_{\phi} \in A$ and there exists $g \in S^{*}$

$$
\left|\frac{z K_{\phi}(z)}{g(z)}-1\right|<1, z \in E .
$$

It is clear that $S_{\mu}^{*} \subset K_{\mu} \subset A$, where $K_{\mu}$ represents the natural close-to-convex analogue of $S_{\mu}^{*}$.

\section{Preliminaries and Lemmas}

Let $\Omega$ denote the class of analytic function of the form

$$
w(z)=\sum_{k=1}^{\infty} w_{k} z^{k}
$$


which satisfy $w(0)=0$ and $|w|<1$ for $z \in E$. Class of such functions is called Scharwz function if $w \in A$. For $p \in P$ given by $(1.2)$, let $p(z)=\frac{1+w(z)}{1-w(z)}$ for some $w \in \Omega$, where $A$ is given by (1.1); on equating coefficients we obtain

$$
p_{1}=2 w_{1}, p_{2}=2 w_{2}+2 w_{1}^{2} .
$$

The following lemmas shall be tools for the present investigation.

Lemma 1. [10] Let $w \in \Omega$ be given by (2.1). Then

$$
\begin{gathered}
\left|w_{2 k-1}\right| \leq 1-\left|w_{1}\right|^{2}-\left|w_{2}\right|^{2}-\left|w_{3}\right|^{2}-\ldots-\left|w_{k}\right|^{2} \text { for } k=2,3, \ldots \\
\left|w_{2 k}\right| \leq 1-\left|w_{1}\right|^{2}-\left|w_{2}\right|^{2}-\left|w_{3}\right|^{2}-\ldots-\left|w_{k}-1\right|^{2}-\left|w_{k}\right|^{2} \text { for } k=1,2,3, \ldots
\end{gathered}
$$

Lemma 2. [10] Let $w \in \Omega$ be given by 2.1. If $\mu \in C$, then

$$
\left|w_{2}-\mu w_{1}^{2}\right| \leq \max \{1,|\mu|\} .
$$

By $(2.2)$ and 2.3 we have the following

Lemma 3. [10] Let $p \in P$ be given by (1.2). Then for $\mu \in C$,

$$
\left|p_{2}-\mu p_{1}^{2}\right| \leq 2-\max \{1,|2 \mu-1|\} .
$$

The inequality is sharp for each complex $\mu$.

Lemma 4. [10] Let $g \in S^{*}$ be given by

$$
g(z)=z+\sum_{k=2}^{\infty} b_{k} z^{k}
$$

Then for any $\mu \in C$,

$$
\begin{gathered}
\left|b_{3}-\mu b_{2}^{2}\right| \leq \max \{1,|4 \mu-3|\} \\
\left|b_{3}-\mu b_{2}^{2}\right| \leq 1+(|4 \mu-3|-1) \frac{\left|b_{2}\right|^{2}}{4},
\end{gathered}
$$

both inequalities are sharp.

Recently Allu et al. [10] provided means by which the result in $S_{\mu}^{*}$ can be extended to $K_{\mu}$. Following their method we give the following results due to generalized discrete probability distribution. 


\section{Coefficient bounds for class $K_{\mu}$}

Theorem 1. Let $K_{\phi} \in K_{\mu}$ be given by 1.3 . Then

$$
\begin{gathered}
\left|\frac{a_{1}}{S}\right| \leq \frac{3}{2}, \\
\left|\frac{a_{2}}{S}\right| \leq \frac{5}{3}, \\
\left|\frac{a_{3}}{S}\right| \leq 1.8443, \\
\left|\frac{a_{4}}{S}\right| \leq 2.00 .
\end{gathered}
$$

Proof. We let

$$
z K_{\phi}^{\prime}(z)=g(z)(1+w(z))
$$

for some $g \in S^{*}$ and some $w \in \Omega$, on equating coefficients in (3.5) and using both (2.1) and (3.5), we obtain

$$
\begin{gathered}
2 \frac{a_{1}}{S}=b_{2}+w_{1} \\
3 \frac{a_{2}}{S}=b_{3}+b_{2} w_{1}+w_{2} \\
4 \frac{a_{3}}{S}=b_{4}+b_{3} w_{1}+b_{2} w_{2}+w_{3} \\
5 \frac{a_{4}}{S}=b_{5}+b_{4} w_{1}+b_{3} w_{2}+b_{2} w_{3}+w_{4} \\
6 \frac{a_{5}}{S}=b_{6}+b_{5} w_{1}+b_{4} w_{2}+b_{3} w_{3}+b_{2} w_{4}+w_{5}
\end{gathered}
$$

where $\left|b_{k}\right| \leq k$ and $\left|w_{k}\right| \leq 1$ for $k \geq 1$. Therefore 3.6 yields

$$
2\left|\frac{a_{1}}{S}\right| \leq\left|b_{2}\right|+\left|w_{1}\right|, \Rightarrow 2\left|\frac{a_{1}}{S}\right| \leq 3 .
$$

Now we let $x_{1}=\left|w_{1}\right|, x_{2}=\left|w_{2}\right|, x_{3}=\left|w_{3}\right|, x_{4}=\left|w_{4}\right|$ from (3.7), we have

$$
3\left|\frac{a_{2}}{S}\right| \leq\left|b_{3}\right|+\left|b_{2}\right|\left|w_{1}\right|+\left|w_{2}\right|
$$


such that Lemma 1 implies

$$
3\left|\frac{a_{2}}{S}\right| \leq 3+2\left|w_{1}\right|+\left(1-\left|w_{1}\right|^{2}\right) \leq 5
$$

since $0 \leq 4+2 x_{1}-x_{1}^{2} \leq 5$ for $x_{1} \in[0,1]$, then $\left|\frac{a_{2}}{S}\right| \leq \frac{5}{3}$.

Furthermore, from 3.8 and Lemma 1

$$
4\left|\frac{a_{3}}{S}\right| \leq\left|b_{4}\right|+\left|b_{3}\right|\left|w_{1}\right|+\left|b_{2}\right|\left|w_{2}\right|+\left|w_{3}\right| \leq 4+3 x_{1}+2 x_{2}+x_{3} .
$$

For $0 \leq x_{1} \leq 1, x_{2} \leq 1-x_{1}^{2}, x_{3} \leq 1-x_{1}^{2}-x_{2}^{2}$, we need to find $\max _{H} g\left(x_{1}, x_{2}, x_{3}\right)$ where $g\left(x_{1}, x_{2}, x_{3}\right)=4+3 x_{1}+2 x_{2}+x_{3}$ and

$$
H\left\{\left(x_{1}, x_{2}, x_{3}\right):=x_{1} \leq 1, x_{2} \leq 1-x_{1}^{2}, x_{3} \leq 1-x_{1}^{2}-x_{2}^{2}\right\} .
$$

It is obvious that the maximum over $H$ occurs on the boundary $\delta H$ which we now consider.

Suppose $x_{3}=1-x_{1}^{2}-x_{2}^{2}$ and $x_{2}=1-x_{1}^{2}$, then

$$
g\left(x_{1}, x_{2}, x_{3}\right)=6+3 x_{1}-x_{1}^{2}-x_{1}^{4}, 0 \leq x \leq 1 .
$$

Solving the above equation using Wolfram Alpha

$$
\max \left\{6+3 x_{1}-x_{1}^{2}-x_{1}^{4}: 0 \leq x_{1} \leq 1\right\}=7.3731 \ldots \quad \text { at } x_{1}=0.72808,
$$

where

$$
\begin{gathered}
7.3731=\frac{1}{24}\left\{148-\frac{968}{\sqrt[3]{54181+2259 \sqrt{753}}}\right\}+\sqrt[3]{54181+2259 \sqrt{753}} \\
0.72808=\frac{\sqrt[3]{27+\sqrt{753}}}{2 \sqrt{39}}-\frac{1}{\sqrt[3]{3(27+\sqrt{753)}}} .
\end{gathered}
$$

Hence $\left|\frac{a_{3}}{S}\right| \leq \frac{1}{4} \times 7.3731=1.8443$. Applying the same method, result for $\frac{a_{4}}{S}$ can be obtained. There is a backward shift between our result and that of [10]. Furthermore, our results established more connections between statistics and univalent functions and geometric function theory and it converges faster. 
Theorem 2. Let $K_{\phi} \in K_{\mu}$ be given by (1.3) and $\mu \in R$.

$$
\begin{gathered}
\text { For } \mu \leq 0,\left|\frac{a_{2}}{S}-\mu \frac{a_{1}^{2}}{S^{2}}\right| \leq \frac{5}{3}-\frac{9}{4} \mu . \\
\text { For } 0 \leq \mu \leq \frac{2}{3}, \quad\left|\frac{a_{2}}{S}-\mu \frac{a_{1}^{2}}{S^{2}}\right| \leq \frac{2\left(10-18 \mu-9 \mu^{2}\right)}{3(4-3 \mu)} .
\end{gathered}
$$

Proof. Since $K_{\phi} \in K_{\mu}$, it is possible to write

$$
z K_{\phi}^{\prime}(z)=g(z) \frac{2 p(z)}{1+p(z)} .
$$

Let $p \in P$ be as defined in $(1.2)$ and $g \in S^{*}$, equating (3.14) using (1.2) and (2.4), we obtain

$$
\begin{aligned}
& \frac{a_{2}}{S}-\mu \frac{a_{1}^{2}}{S^{2}}=\frac{1}{3}\left(b_{3}-\frac{3 \mu b_{2}^{2}}{4}\right)+\frac{b_{2} p_{1}}{12}(2-3 \mu)+\frac{1}{6}\left(p_{2}-\frac{p_{1}^{2}}{2}\right)-\frac{\mu p_{1}^{2}}{16} . \\
& \frac{a_{2}}{S}-\mu \frac{a_{1}^{2}}{S^{2}}=\frac{1}{3}\left(b_{3}-\frac{3 \mu b_{2}^{2}}{4}\right)+\frac{b_{2} p_{1}}{12}(2-3 \mu)+\frac{1}{6}\left(p_{2}-\frac{4+3 \mu}{8} p_{1}^{2}\right) .
\end{aligned}
$$

The conditions in Theorem 2 above are treated as follows:

Case in (3.12): For $\mu \leq 0$ using (3.15) with $x=\left|p_{1}\right|$ and applying Lemma 3 and Lemma 4 with $\left|b_{2}\right| \leq 2$ we have

$$
\begin{gathered}
=\left|\frac{1}{3}\left(b_{3}-\frac{3 \mu b_{2}^{2}}{4}\right)+\frac{a_{2}}{S}-\mu \frac{a_{1}^{2}}{S^{2}}\right| \\
\leq \frac{b_{1}}{12}(2-3 \mu)+\frac{1}{6}\left(p_{2}-\frac{p_{1}^{2}}{2}\right)-\mu \frac{p_{1}^{2}}{16} \mid \\
=\frac{1}{3}\left(3 \mu-3\left|+\frac{1}{6}\right| 2-3 \mu\left|x+\frac{1}{6}\right| 2-\frac{x^{2}}{2} \mid-\frac{1}{16} \mu x^{2}\right.
\end{gathered}
$$

where $x \in[0,2]$. Since RHS of $(3.19)$ increases with respect to $x \in[0,2]$,

$$
\left|\frac{a_{2}}{S}-\mu \frac{a_{1}^{2}}{S^{2}}\right| \leq\left[\frac{1}{3}(3 \mu-3)+\frac{1}{6}(2-3 \mu) x+\frac{1}{6}\left(2-\frac{x^{2}}{2}\right)-\frac{1}{16} \mu x^{2}\right]_{x=2}=\frac{5}{3}-\frac{9 \mu}{4} .
$$


Case in (3.13): $0 \leq \mu \leq \frac{2}{3}$, we again use 3.15 with $x=\left|p_{1}\right|$, which gives

$$
\left|\frac{a_{2}}{S}-\mu \frac{a_{1}^{2}}{S^{2}}\right| \leq\left[\frac{1}{3}(3 \mu-3)+\frac{1}{6}(2-3 \mu) x+\frac{1}{6}\left(2-\frac{x^{2}}{2}\right)+\frac{1}{16} \mu x^{2}\right] .
$$

The above equation has maximum value at $x=\frac{4(3 \mu-2)}{(3 \mu-4)} \in[0,2]$ so the bound for $0 \leq \mu \leq \frac{2}{3}$.

The results in Theorem 1 and Theorem 2, further strengthen the connections between statistics and geometric function theory.

For special values of $a_{k-1}$ various well known discrete probability distribution such as Yule-Simeon distribution, logarithmic distribution, Poisson distribution, Binomial distribution, Beta-Binomial distribution, Zeta distribution, geometric distribution and Bernoulli distribution can be obtained.

In the next section, we shall consider the Poisson distribution.

\section{Coefficients of Poisson Distribution}

Recently, Porwal in [8] introduced a power series whose coefficients are probability of Poisson distribution as follows

$$
K_{m}(z)=z+\sum_{k=2}^{\infty} \frac{m^{k-1}}{(n-1) !} e^{-m} z^{k} .
$$

By ratio test the radius of convergence of the series 4.1 is infinity, $m$ is called the parameter and $m>0$. Using the above the author obtained some interesting results on classes of analytic functions (see also [3] and the relevance references therein.

The Poisson distribution is a tool used in probability theory statistics to predict the amount of variation from a known average of occurrence, within a given time. That is to determine how much variation there will likely be from that average number of occurrence and also determine the probable maximum and minimum number of times the event will occur within the specified time frame. Company can also use the Poisson distribution to examine how they may be able to take steps to improve their operational efficiency. 
We state the following:

Theorem 3. Let $K_{m} \in K_{\mu}$ be given by 4.1) $m>0$. Then

$$
m e^{-m} \leq \frac{3}{2}, m^{2} e^{-m} \leq \frac{10}{3}, m^{3} e^{-m} \leq 11.597 .
$$

Proof. Let us write

$$
z K_{m}^{\prime}(z)=g(z)(1+w(z))
$$

where $K_{m}(z)$ is as given in 4.1 and $g(z)=z+\sum_{k=2}^{\infty} b_{k} z^{k}$ for $\omega \in \Omega$. Equating the coefficients in 4.2 the result follows.

Theorem 4. Let $K_{m} \in K_{\mu}$ be given by 4.1. Then

$$
\begin{gathered}
\text { for } \mu \leq 0, \quad m^{2} e^{-m}\left(1-\mu e^{-m}\right) \leq \frac{2}{3}-\frac{7 \mu}{4} \\
\text { for } \mu \leq \frac{2}{3}, \quad m^{2} e^{-m}\left(1-\mu e^{-m}\right) \leq \frac{2\left(12 \mu-3 \mu^{2}-8\right)}{3 \mu-8} .
\end{gathered}
$$

Proof. The method of proof is the same as that for Theorem 2 and also for the two cases considered except that the expression considered for the second case has a maximum value at $x=\frac{4(3 \mu-4)}{3 \mu-8}$ in $[0,2]$, so bound for $\mu \leq \frac{2}{3}$ follows. With various choices of the value $m$, many interesting results can be obtained.

\section{Conclusion}

Probability and Statistics play a vital role in every field of human activity, in particular, they are quantitative tools widely used in the area of economics and finance. With various choices of values for parametric $m$ and $a_{k-1}$ many interesting decision can be taking, simply because any series of actions will generate different results depending on variety of circumstances. Also from economics point of view our results shall be useful in the decision making processes. 


\section{References}

[1] Abiodun Tinuoye Oladipo, Generalized distribution associated with univalent functions in conical domain, An. Univ. Oradea Fasc. Mat. XXVI(1) (2019), 161-167.

[2] Abiodun Tinuoye Oladipo, Bounds for probabilities of the generalized distribution defined by generalized polylogarithm, Punjab Univ. J. Math. 51(7) (2019), 19-26.

[3] Abiodun Tinuoye Oladipo, Bounds for Poisson and neutrosophic Poisson distributions associated with Chebyshev polynomial, Palestine J. Math. (to appear).

[4] A. W. Goodman, Univalent Functions, Texts in Pure Mathematics, Volume I, Mariner Publishing Co., Tampa, FL, 1983.

[5] A. W. Goodman, Univalent Functions, Texts in Pure Mathematics, Volume II, Mariner Publishing Co., Tampa, FL, 1983.

[6] F. R. Keogh and E. P. Merkes, A coefficient inequality for certain classes of analytic functions, Proc. Amer. Math. Soc. 20(1) (1969), 8-12.

[7] Saurabh Porwal, Generalized distribution and its geometric properties associated with univalent functions, Journal of Complex Analysis 2018 (2018), Art. ID 8654506, 5 pp. https://doi.org/10.1155/2018/8654505

[8] Saurabh Porwal, An application of a Poisson distribution series on certain analytic functions, Journal of Complex Analysis 2014 (2014), Art. ID 934135, 3 pp. http://dx.doi.org/10.1155/2014/984135

[9] R. Singh, On a class of star-like functions, Compos. Math. 19(1) (1968), 78-82.

[10] A. Vasudevarao, J. Sokol and Derek K. Thomas, On a close-to-convex analogue of certain starlike functions, Bull. Aust. Math. Soc. 102(2) (2020), 268-281. https://doi.org/10.1017/S0004972719001606

This is an open access article distributed under the terms of the Creative Commons Attribution License (http://creativecommons.org/licenses/by/4.0/), which permits unrestricted, use, distribution and reproduction in any medium, or format for any purpose, even commercially provided the work is properly cited. 